Questions vives

\section{Questions Vives}

Recherches en éducation

$N^{\circ} 31 \mid 2019$

Évaluation et numérique : Nouvelles pratiques?

Nouvelles opportunités?

\title{
Évaluation orientée vers la maîtrise ou évaluation orientée vers la performance? Des contextes qui régulent les performances des élèves lors d'un apprentissage
}

Should assessment be mastery oriented or performance oriented? A study of the contexts regulating pupil achievement in a learning sequence.

Carine Souchal et Marie-Christine Toczek

\section{(2) OpenEdition}

\section{Édition électronique}

URL : http://journals.openedition.org/questionsvives/4063

DOI : 10.4000/questionsvives.4063

ISSN : $1775-433 \mathrm{X}$

Éditeur

Université Aix-Marseille (AMU)

\section{Édition imprimée}

Date de publication : 30 juillet 2019

ISBN : 978-2-912643-55-1

ISSN : 1635-4079

\section{Référence électronique}

Carine Souchal et Marie-Christine Toczek, «Évaluation orientée vers la maîtrise ou évaluation orientée vers la performance? Des contextes qui régulent les performances des élèves lors d'un apprentissage », Questions Vives [En ligne], N 31 | 2019, mis en ligne le 30 août 2020, consulté le 03 avril 2021. URL : http://journals.openedition.org/questionsvives/4063 ; DOI : https://doi.org/10.4000/ questionsvives.4063

Ce document a été généré automatiquement le 3 avril 2021.

\section{(†)

Questions Vives est mis à disposition selon les termes de la licence Creative Commons Attribution Pas d'Utilisation Commerciale - Pas de Modification 4.0 International. 


\section{Évaluation orientée vers la maîtrise ou évaluation orientée vers la performance? Des contextes qui régulent les performances des élèves lors d'un apprentissage}

Should assessment be mastery oriented or performance oriented? A study of the contexts regulating pupil achievement in a learning sequence.

Carine Souchal et Marie-Christine Toczek

\section{INTRODUCTION}

Faut-il noter les élèves? Cette question est de manière récurrente posée par les différents acteurs gravitant autour de l'école, et ce, dans plusieurs pays de l'OCDE (Mons, Toczek, West, Barkous, 2014). Elle prend très souvent la forme de véritables polémiques où sein desquelles de multiples arguments, pour ou contre, sont avancés. Comme le mentionne le rapport de l'Inspection Générale de l'Education Nationale (2013), ces arguments sont très rarement des arguments scientifiques, mais tiennent plutôt à des opinions ou idéologies. L'objectif de ce travail est de mettre en place une étude qui a la volonté d'établir des liens de cause à effet entre des contextes évaluatifs et des performances d'élèves. De ce fait, dans la revue de littérature qui suit, nous avons sélectionné des études relevant de méthodologies quantitatives en privilégiant des travaux qui cherchaient à établir des liens de cause à effet. 


\section{DOUBLE FONCTION DU SYSTEME SCOLAIRE}

2 En effet, selon plusieurs chercheurs (e.g., Dubet \& Martucelli, 1988 ; Butera, Buchs, \& Darnon, 2011) les systèmes scolaires assurent plusieurs fonctions: on attend d'eux qu'ils éduquent et socialisent les élèves, mais ils assurent également une fonction de distribution (Darnon, Dompnier, \& Poortvliet, 2012). Ils distribuent en effet des diplômes et par ce biais assignent aux individus une place plus ou moins élevée au sein de la société (Duru-Bellat, 2009). Selon Dubet et Martucelli (1988), cette dernière fonction, jadis soumise aux deux premières, a pris, avec la massification du public scolaire et l'ouverture de l'école à tous, un poids croissant. De nos jours, l'importance de cette fonction distributive aurait ainsi surpassé celle des deux autres fonctions: l'enjeu de la réussite scolaire serait donc devenu la réussite sociale, et parallèlement la notion d'échec scolaire serait intimement liée à celle d'échec social. L'école a donc à conjuguer quotidiennement plusieurs objectifs qui peuvent paraître paradoxaux : d'un côté, on lui demande de former, de faire progresser les élèves dont elle a la charge, mais de l'autre, elle doit également les classer, les hiérarchiser, afin de remplir son rôle de sélection. L'objectif de cette recherche est d'étudier et de comparer les effets de ces deux types d'injonctions sur les performances des élèves. Pour cela, elle centre son attention sur l'un des outils utilisés quotidiennement par les professeurs pour servir ces deux fonctions : l'évaluation.

\section{DIFFERENTS TYPES D'EVALUATION}

3 L'évaluation des élèves est l'un des outils dont dispose l'Ecole pour mener à bien ses différentes missions. En effet, évaluer les élèves, c'est assurer une régulation (Crahay, 1996; Hadji, 2015). Mais cette régulation peut-être elle-même de différents types, répondant chacun à l'une des fonctions des systèmes éducatifs cités en amont : ainsi lorsque l'évaluation revêt une fonction de régulation sociale, elle vise à s'assurer que les élèves répondent bien aux exigences imposées par le système dans lequel ils sont placés. C'est ce genre d'évaluation qui permet de décerner un diplôme, de procéder à une orientation ou qui permet de décider du passage dans le niveau supérieur. L'évaluation peut également intervenir en fin d'une séquence d'apprentissage ou d'une période scolaire plus ou moins longue. Elle porte alors le nom d'évaluation sommative et correspond à la fonction distributive que doit servir l'Ecole. Le second type de régulation que peut assurer l'évaluation est la régulation pédagogique: son but est alors de s'assurer que le système d'enseignement répond bien aux besoins des élèves et qu'il leur permet d'apprendre. Il s'agit alors de donner des informations aux enseignants afin que l'enseignement délivré soit adapté aux élèves et à leurs besoins. Son but est également d'aider l'élève à se situer dans le processus d'apprentissage (Hattie \& Timperley, 2007). Cette évaluation porte le nom d'évaluation formative, on peut la rapprocher de la fonction formative assignée à l'Ecole.

4 Dans les classes, pour évaluer leurs élèves, les enseignants ont à leur disposition un panel d'outils qui permettent d'assurer ces deux fonctions régulatrices. Notons que ces outils peuvent tous servir l'une ou l'autre des fonctions de régulation qui viennent d'être présentées. Mais ces différents dispositifs évaluatifs ont-ils une incidence sur le type de motivation ressenti par les élèves qui les rencontrent? 


\section{EVALUATION ET TYPES DE MOTIVATION}

\section{L'étude des climats motivationnels}

5 Les pratiques des enseignants au sein des classes sont en effet connues pour induire chez les élèves certains types de motivation, qui, en retour, ont une influence sur les performances et les apprentissages de ces élèves (l'annexe 1 présente un tableau synthétique des travaux présentés ici). On a ainsi pu identifier des «climats motivationnels » découlant de ces pratiques et étant plutôt orientés vers la maîtrise des savoirs (climat de maîtrise) ou alors plutôt vers la production de performances (climat de compétition). En fait, ces climats semblent pouvoir être définis par les choix effectués par les enseignants sur six variables, répertoriées par Epstein (1988) et nommées TARGET. Ainsi, selon cet auteur, le climat instauré en classe découle du type de Tâches proposé aux élèves (T), de la façon de gérer l'Autorité (A), du type de Reconnaissance dont jouissent les élèves (R), de la façon de travailler en Groupes habituellement utilisée en classe (G), du type d'Evaluation proposé (E) et enfin de la façon dont est géré le temps en classe $(\mathrm{T})$.

6 Ames et ses collaborateurs (Ames, 1992 a et b ; Ames \& Ames, 1984 ; Ames \& Archer, 1988 ; Ames \& Maehr, 1989) ont essayé de décrire plus finement le type d'arrangement de ces six variables qui pouvaient caractériser un climat de maîtrise et un climat de compétition. Leurs conclusions montrent que le climat de maîtrise est ainsi décrit comme encourageant l'autonomie, la coopération entre les individus et orientant leur attention sur le processus d'apprentissage. Le climat de compétition serait, quant à lui, défini comme suscitant la compétition entre les individus, en accordant une grande importance aux notes obtenues. Il les amènerait donc à considérer le résultat final, la performance produite comme l'élément le plus important.

7 Le caractère plus ou moins favorable aux apprentissages de ces différents climats a également été investigué. Ainsi, on sait que le climat orienté vers la maîtrise est un climat qui est généralement relié à une plus grande poursuite de buts de maîtrise de la part des élèves (Anderman \& Midgley, 1997 ; Meece, Anderman \& Anderman, 2006) et à un plus haut niveau de motivation intrinsèque (Cury et al., 1996). Ce climat semble également corrélé avec l'utilisation de stratégies d'apprentissage adaptées et efficaces (Ames \& Archer, 1988) ainsi qu'avec l'obtention de meilleures performances scolaires (Midgley \& Urdan, 2001) même si cet effet n'est pas toujours obtenu (Anderman \& Midgley, 1997). Les effets du climat orienté vers la performance ne sont, quant à eux, pas aussi clairs: ils sont souvent associés à la poursuite de buts de performance (Ntoumanis, 2001), à l'emploi de stratégies inadaptées comme l'auto-handicap ou l'évitement à rechercher de l'aide (Midgley \& Urdan, 2001) ou encore au manque d'engagement dans des tâches difficiles (Sarrazin, Famose \& Cury, 1995). Le recours à la tricherie semble également associé à ce type de climat (Anderman, Griesinger \& Westerfield, 1998). Cependant, concernant les performances des élèves Selon les études, soit ce contexte n'a pas d'effet sur les performances des élèves, soit il peut leur être favorable (Midgley \& Urdan, 1995).

8 De tous les facteurs situationnels influençant le climat motivationnel, Ames (1992b) décrit le processus d'évaluation de la tâche comme étant le plus important. Plusieurs études isolant ce facteur montrent en effet que, selon ses caractéristiques, une situation évaluative peut être reliée à l'adoption de patterns motivationnels différents. 


\section{L'objectif annoncé de la tâche évaluative}

Kaplan et Maehr (2007) recensent de nombreuses études en ce sens. Ces auteurs notent que les inductions concernent souvent l'objectif annoncé de la tâche proposée aux participants de l'étude. Ainsi, les inductions censées induire un climat orienté cers la maitrise présentent souvent la tâche comme un support d'apprentissage et de progrès, avec en point de mire un objectif d'évolution personnelle. En revanche, cette même tâche est présentée comme un moyen de démontrer ses capacités lorsqu'il s'agit d'orienter la tâche vers la production de performances. Ainsi, Elliot et Dweck (1988) proposent de renforcer l'adhésion aux buts de maîtrise en expliquant à leurs participants que la tâche réalisée les aidera dans leurs études. Pour susciter l'adhésion aux buts de performance, ces mêmes auteurs disent que les performances réalisées sur cette même tâche seront filmées et évaluées normativement. De la même manière, Butler (1993) propose une tâche qui permettra aux participants soit d'exercer leurs capacités à résoudre des problèmes et de repérer leurs stratégies inefficaces en vue de les améliorer (induction de buts de maîtrise), soit de tester ces capacités à la résolution de problèmes (induction de buts de performance).

\section{La nature du feed-back}

10 La nature du feed-back évaluatif donné aux étudiants a été également particulièrement étudiée. Ainsi, Ames (1984) remarque que les situations qui rendent saillantes la comparaison sociale affectent particulièrement la motivation des élèves et les conduisent à orienter leur attention vers leurs performances et vers la répartition des performances des autres. Ames (1992b) note à ce sujet que les évaluations qui amènent les individus à situer leurs performances actuelles au sein de leurs performances antérieures (comparaison intra-temporelle) sont plus favorables à l'adoption de buts de maîtrise que les évaluations normatives qui conduisent, elles, plutôt à comparer les performances actuelles aux performances des autres individus évalués. De la même manière Butler et Nissan (1986) étudient les effets de différentes natures de feed-back donnés sur les buts adoptés par les individus. Elles distribuent ainsi soit des renforcements appelés task oriented feed-back renseignant plutôt sur le degré de maîtrise des savoirs, soit des renforcements ego oriented, c'est-à-dire une note reflétant le niveau de performance. Ces auteurs montrent que ce sont les task oriented feed-back qui ont les meilleures conséquences en termes motivationnels et de performance. De la même manière, Butler (1987) fait varier la nature du feed-back évaluatif en attribuant soit une note (évaluation normative, ego-oriented soit un commentaire écrit, taskoriented) à des étudiants. Puis, elle interroge ces étudiants sur leurs préoccupations lors de la passation de la tâche. Les étudiants ayant reçu une note déclarent avoir été plus préoccupés par le fait de vouloir démontrer leurs capacités que les étudiants ayant reçu un commentaire, qui, en revanche, rapportent une plus grande volonté de tirer partie de cette tâche pour apprendre.

11 On le voit donc, le type d'évaluation annoncé aux individus rend plus ou moins saillants certains types de buts. En fait, les différents types de buts de réussite adoptés par les individus diffèrent par le standard de comparaison choisi par ces individus : alors que les buts de performance reposent sur de la comparaison sociale en posant les performances de l'individu par rapport aux autres, les buts de maîtrise quant à eux 
semblent plutôt reposer sur un processus de comparaison temporelle, situant les performances de l'individu par rapport à celles déjà observées (Ames, 1992a , 1992b ; Butler, 1987 ; Kaplan \& Maehr, 2007). Il n'est donc pas étonnant qu'en fonction des types de comparaisons suggérés par les résultats des évaluations, les individus puissent modifier leurs buts de réussite avec les conséquences que l'on connaît sur leurs performances et leur apprentissage.

12 La présente étude se proposait de tester ce lien entre types de comparaisons suggérés, buts adoptés et performances réalisées. Cette étude a été réalisée auprès d'enfants âgés de 9ans et placés dans une situation d'apprentissage en mathématiques. L'idée était de camper, d'un côté une situation évaluative orientée vers la maîtrise des savoirs en question, en amenant les élèves à comparer leurs performances à leurs performances antérieures et de l'autre, une situation évaluative orientée vers la performance, en incitant les élèves à comparer leurs propres performances à celles des autres élèves. Notre hypothèse était que l'apprentissage et les performances des élèves seraient meilleurs en situation évaluative orientée vers la maîtrise. L'originalité de cette étude était que le caractère "orientée vers la performance" de la seconde situation évaluative n'était pas induit, comme souvent, par l'attribution d'une note, mais simplement par un critère de réussite énoncé oralement par l'expérimentateur. De plus, les effets des contextes évaluatifs orientés "maitrise " versus "performance » n'ont, à notre connaissance, jamais été étudiés sur une population d'élèves jeunes.

\section{Objectifs de l'étude}

13 Cette étude a été menée auprès d'élèves âgés de 9 ans ( $4^{e}$ grade). Elle s'est déroulée auprès d'élèves ayant l'habitude d'être évalués par un système de couleur couramment utilisé dans les écoles élémentaires française où on signale en vert une compétence acquise, en orange, une compétence en cours d'acquisition et en rouge une compétence non acquise. De ce fait, l'induction d'un contexte de maîtrise versus de performance s'est faite sans jamais aucun recours à la note, simplement en attirant l'attention des participants sur les critères de réussite: dans l'une des conditions il s'agissait d'un critère lié à une comparaison intrapersonnelle des performances alors que dans l'autre, le critère était lié à une comparaison interpersonnelle des performances. La tâche était une tâche de mathématiques. Pour cette étude, nous adoptons une posture de recherche bien particulière dans le but d'établir des liens de cause à effet entre différents contextes évaluatifs induits et les performances des élèves ainsi que sur la rapidité de leurs apprentissages. En outre, on mesurait également l'effet des différents types d'évaluation sur le type de motivation ainsi générée auprès des élèves. Nous avons donc choisi de suivre une méthode quasi-expérimentale qui nous permettrait d'établir ces liens de cause à effet.

\section{METHODE}

\section{Population}

103 élèves ont participé à cette étude. Sur ce total, les données relatives à 4 élèves ont dû être écartées car ces élèves étaient absents lors de la phase expérimentale. Sur ces 99 participants, 94 ont déclaré la réussite en mathématiques comme importante à très 
importante pour eux. Ces réponses indiquant un niveau d'identification satisfaisant au domaine des mathématiques, c'est sur les données propres à ces 94 participants que les analyses ont été effectuées. 38 étaient des garçons et 56 des filles. 49 participants étaient assignés à la condition orientée vers la maîtrise alors que 45 réalisaient l'étude en condition orientée vers la performance.

\section{Protocole}

Le choix de la méthode quasi-expérimentale nous a conduites à choisir une tâche nonscolaire présentée aux élèves dans une situation "ordinaire ». L'expérimentatrice annonçait aux élèves qu'ils allaient réaliser une tâche de mathématiques qui permettrait de montrer leurs capacités. Cette tâche devait donner lieu à une évaluation selon le dispositif en vigueur dans ces classes : l'obtention de la couleur "verte" signifiant une évaluation positive (la couleur rouge symbolisant une évaluation négative). Elle expliquait alors que la tâche impliquait de mémoriser une figure géométrique pendant quelques secondes, puis de la reproduire de mémoire sans le modèle. Elle précisait qu'il y aurait cinq essais.

Puis l'expérimentatrice annonçait la première induction. Dans la condition orientée vers la maitrise, elle disait aux élèves que l'objectif était d'apprendre et donc qu'ils devaient, pour obtenir "vert», toujours s'améliorer, c'est-à-dire obtenir plus d'éléments justes au second essai qu'au premier, au troisième qu'au second, etc. Dans la condition orientée vers la performance, elle disait en revanche que le but, pour avoir " vert ", était d'être meilleur que les autres, c'est-à-dire d'obtenir à chaque essai, plus d'éléments justes que la moyenne des éléments justes obtenus par les élèves de la classe. Chaque classe était divisée en deux et la moitié recevait l'induction orientée vers la maîtrise alors que l'autre moitié recevait l'induction orientée vers la performance.

Puis l'expérimentatrice montrait la figure pendant 30 secondes. Elle laissait ensuite 3 minutes aux élèves pour reproduire la figure de mémoire. Après rappel de l'induction, elle montrait à nouveau la figure pendant 30 secondes et laissait ensuite 3 minutes aux élèves. Elle répétait ce protocole 5 fois.

A la fin des cinq essais, les enfants devaient choisir une figure géométrique avec laquelle ils aimeraient à nouveau réaliser ce type de tâche, l'une ayant été pré-testée comme paraissant simple et l'autre comme paraissant difficile auprès d'enfants du même âge que ceux composant notre échantillon expérimental.

Afin de garantir des conditions de passation équivalentes pour tous les élèves des groupes, l'attribution du code couleur a été réalisée de manière différée par rapport à la passation. Ce code couleur n'a pas été utilisé par les chercheurs puisqu'il a été annoncé aux élèves dans le simple objectif de contextualiser la tâche au regard des pratiques évaluatives de l'enseignante statutaire. Afin de traiter les résultats, les chercheurs avaient besoin de résultats chiffrés à des fins statistiques. Ces résultats chiffrés ont été obtenus d'après la méthode détaillée ci après. 


\section{Matériel}

\section{La tâche}

20 d'un test visuo-spatial utilisant la figure de Rey-Osterrieth. Ce test consiste à faire reproduire de mémoire une figure géométrique complexe après quelques secondes d'exposition. Les indicateurs de performances étaient les suivants : pour chacun des essais, la production de l'élève était notée selon un barème déterminé par le manuel de cotation du test de la figure de Rey. Chaque élément présent et bien placé recevait la note de 2 , cette note était ramenée à 1 si l'élément était déformé ou mal placé ou à 0.5 s'il était déformé et mal placé. Cela aboutissait à un total dont le maximum était 44 . C'est sur la base de ce score qu'ont été calculées les moyennes présentées dans le tableau 1 et le graphique 1 et qu'ont été réalisées les analyses statistiques.

\section{Mesure de motivation}

21 Cette mesure était inspirée de la mesure de Mueller \& Dweck (1998). Afin de savoir si les élèves étaient plutôt animés d'une motivation orientée vers la maîtrise (apprendre de nouvelles choses) ou d'une motivation orientée vers la performance (réussir mieux que les autres), on leur demandait de choisir avec quelle figure ils préféreraient travailler la fois suivante. Le choix présentait une figure jugée lors d'un pré-test initial comme facile (score moyen de difficulté estimée : 1.2/10) et une figure préalablement jugée difficile (score moyen de difficulté estimée : 8.6/10). Cette mesure sera traitée sous forme d'une comparaison de pourcentages.

\section{Résultats}

\section{Vérification de l'induction expérimentale}

Des $t$-tests ont été lancés sur les réponses des participants. Les individus ayant réalisé la tâche en contexte orienté vers la performance estiment significativement plus que leur travail sera considéré comme réussi s'ils ont fait «mieux que les autres» et s'ils ont dessiné «plus d'éléments justes que les autres élèves » $(\mathrm{M}=4.03$; $\mathrm{ET}=0.89)$ que les élèves ayant travaillé en contexte orienté vers la maîtrise $(\mathrm{M}=3.64 ; \mathrm{ET}=0.89)$ et $t(92)=-2.13, p<.05$.

Parallèlement, les élèves ayant réalisé la tâche en contexte orienté vers la maitrise estiment significativement plus que leur travail sera considéré comme réussi s'ils ont "beaucoup progressé » et s'ils ont dessiné de plus en plus d'éléments justes $(M=4.10$; $\mathrm{ET}=0.92)$ comparativement aux élèves ayant travaillé en contexte orienté vers la performance $(\mathrm{M}=3.73 ; \mathrm{ET}=0.96), t(92)=2.12, p<.05$.

\section{Effet sur les performances}

Cette étude avait pour objectif de comparer les performances mises en œuvre par des élèves âgés de 9 ans placés dans deux contextes évaluatifs différents: un contexte orienté vers la maîtrise grâce à une induction invitant les élèves à comparer leurs résultats avec leurs résultats antérieurs et un contexte orienté vers la performance où l'attention des élèves était centrée sur la comparaison avec les autres élèves. Plus 
précisément, il était attendu que les élèves obtiennent de meilleures performances dans le contexte orienté vers la maîtrise et que leur apprentissage soit plus rapide dans ce contexte.

Afin de mesurer l'impact des inductions évaluatives sur les performances des individus, il a été réalisé une analyse de variance pour mesures appariées avec un plan 2 condition (orientée vers la maîtrise $v s$ orientée vers la performance) $* 5$ (essais). Ces analyses ont donc été lancées sur les scores de performances obtenues aux 5 essais, reportés Tableau 1.

Tableau 1. Moyennes et écarts-types obtenus pour les différents essais en fonction du type d'évaluation.

\begin{tabular}{|c|c|c|c|}
\hline & & Moyennes & Ecarts-types \\
\hline \multirow{2}{*}{ Essai 1} & Condition « maîtrise » : & 7.33 & 5.67 \\
\hline & Condition «performance »: & 5.76 & 3.65 \\
\hline \multirow{2}{*}{ Essai $2 *$} & Condition « maîtrise » : & 13.26 & 7.55 \\
\hline & Condition « performance »: & 9.66 & 5.49 \\
\hline \multirow{2}{*}{ Essai $3^{* *}$} & Condition « maîtrise » : & 17.16 & 8.77 \\
\hline & Condition « performance »: & 13.14 & 6.89 \\
\hline \multirow{2}{*}{ Essai $4^{* * *}$} & Condition « maîtrise »: & 22.84 & 9.35 \\
\hline & Condition « performance $»:$ & 15.83 & 7.39 \\
\hline \multirow{2}{*}{ Essai $5^{* * *}$} & Condition « maîtrise » : & 27.02 & 10.21 \\
\hline & Condition « performance »: & 19.55 & 8.41 \\
\hline
\end{tabular}

Les différences sont significatives à ${ }^{*} p<.05,{ }^{* \star} p<.01,{ }^{* \star *} \mathrm{p}<.001$

Indication de lecture : A l'essai 1, la moyenne des scores obtenus par les élèves placés en condition " évaluation orientée vers la maîtrise » est de 7.33 (sur un total possible de 44 points). La moyenne des scores obtenus par les élèves placés en condition «évaluation orientée vers la performance » est de 5.76. La différence entre ces deux scores n'est pas significative.

Cette analyse révèle tout d'abord un effet principal du temps de passation $F(4,97)=296.16, p<.000, \eta^{2}=.75$. Le test de contraste mené sur ces données révèle une tendance linéaire $F(1,97)=525.4, p<.000, \eta^{2}=.84$ révélant ainsi que les performances s'améliorent au fil des essais.

L'analyse de variance montre également un effet principal du contexte évaluatif. Conformément aux hypothèses, les individus placés dans la condition évaluative orientée vers la maîtrise réussissent globalement mieux $(\mathrm{M}=17.52$, $\mathrm{ET}=0.95)$ que les individus placés dans la condition évaluative orientée vers la performance $(M=12.79$, $\mathrm{ET}=0.98), F(1,97)=12.57, p<.001, \eta^{2}=.12$. Un test d'effet simple avec ajustement de Bonferonni révèle que cette différence de performance entre les deux conditions se retrouve pour chacun des essais, excepté l'essai numéro 1 pour lequel la différence n'est pas significative.

On note également un effet d'interaction entre la condition évaluative et les essais successifs $F(4,97)=10.30, p<.001, \eta^{2}=.1$. L'analyse des moyennes reportées Figure 1 montre que cette interaction est due à un creusement des écarts entre les 
performances des individus placés dans les deux conditions évaluatives en fonction du nombre d'essais réalisés : les individus apprennent plus et plus vite lorsqu'ils sont placés dans la condition évaluative orientée vers la maîtrise que lorsqu'ils sont placés dans la condition évaluative orientée vers la performance.

Figure 1 : Performances obtenues aux différents essais en fonction du type d'évaluation prodigué.

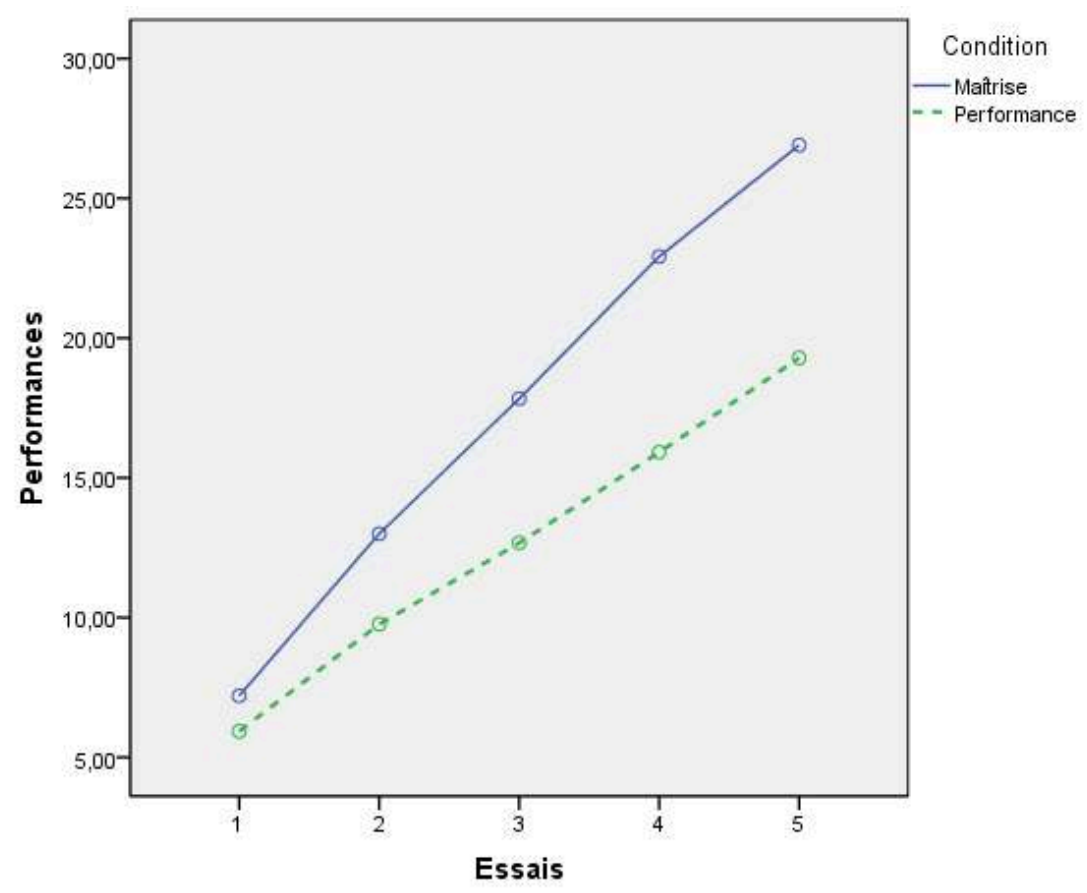

\section{Type de motivation engendrée}

Nous attendions que les participants de la condition évaluative orientée vers la maitrise choisissent plus que les autres l'exercice «difficile». Les participants de la condition orientée vers la performance devaient, quant à eux, préférer choisir l'exercice plus facile.

Un test du Khi-deux d'indépendance a été réalisé sur nos données. Ce test est significatif, indiquant que les choix effectués dans les deux conditions ne peuvent pas être considérés comme également répartis. Le Tableau 2 et la Figure 2 montrent que les participants dont l'attention avait été orientée vers la maîtrise choisissent en majorité la figure la plus facile $(66.7 \%)$ alors que les participants dont l'attention avait été orientée vers la performance choisissent en majorité la figure difficile (71.4\%), $\chi^{2}(1)=10.94, p<.001$.

Tableau 2 : Pourcentage des élèves de chaque condition ayant choisi la figure facile et la figure difficile comme prochain exercice.

\begin{tabular}{|l|l|l|}
\hline & Maîtrise & Performance \\
\hline Figure facile & $28.6 \%$ & $66.7 \%$ \\
\hline
\end{tabular}




\begin{tabular}{|l|l|l|}
\hline Figure difficile & $\mathbf{7 1 . 4} \%$ & $33.3 \%$ \\
\hline TOTAL & $100 \%$ & $100 \%$ \\
\hline
\end{tabular}

Figure 2 : Pourcentage des élèves de chaque condition ayant choisi la figure facile et la figure difficile comme prochain exercice

Indication de lecture : $28.6 \%$ des élèves placés en condition d'évaluation orientée vers la maîtrise ont choisi une figure facile comme exercice ultérieur. Les autres élèves de cette condition (71.4\%) ont choisis une figure compliquée.

\section{DISCUSSION}

31 Cette étude avait pour objectif de comparer l'effet de deux conditions évaluatives sur les performances des élèves lors d'une tâche d'apprentissage en mathématiques. Pour cela, des élèves âgés de 9 ans étaient confrontés à une situation d'apprentissage en 5 essais d'une figure géométrique. Ils étaient prévenus que leurs performances seraient évaluées à l'issue de ces 5 essais. Dans la condition orientée vers la maîtrise, l'attention des élèves était portée sur leur propre progression puisqu'on leur disait que leur évaluation serait positive si leurs performances s'amélioraient au cours des 5 essais. Dans la condition orientée vers la performance, l'attention des élèves était centrée sur leurs résultats puisqu'on leur expliquait que leur évaluation serait positive si, à chaque essai, ils faisaient mieux que la moyenne de la classe. Il était attendu que la condition orientée vers la maitrise, en activant chez les élèves une orientation motivationnelle elle-même orientée vers la maîtrise, soit plus bénéfique aux apprentissages et aux performances que la condition orientée vers la performance.

Cette étude présentait tout d'abord quelques limites théoriques et opérationnelles: l'échantillon expérimental n'était pas d'une grande amplitude et les participants appartenaient tous à des écoles rurales et semi-rurales posant ainsi la question de la généralisation de nos résultats. De plus, la tâche utilisée avait été choisie car sa réalisation ne nécessitait pas de pré-requis. L'idée était alors de n'observer que l'effet situationnel des inductions en s'affranchissant le plus possible des différences de niveaux scolaires des élèves pouvant éventuellement préexister. La contrepartie de ce choix est une diminution de la validité écologique de cette étude, la tâche choisie n'étant assurément pas une tâche d'apprentissage scolaire. Malgré ces limites, cette étude présente des résultats intéressants allant dans le sens des hypothèses.

\section{Conditions évaluatives, apprentissage, performances et motivation}

33 En effet, dans cette étude, les élèves placés en condition évaluative orientée vers la maîtrise obtiennent de meilleurs résultats et progressent plus que les élèves placés en condition orientée vers la performance. De plus, cette condition semble avoir un effet bénéfique sur le type de motivation ressentie ensuite par les élèves : ceux-ci choisissent également majoritairement de travailler plus tard sur une tâche paraissant pourtant très complexe. Ce choix semble alors trahir leur intérêt pour des tâches complexes leur permettant d'acquérir de nouvelles connaissances sans pour autant leur garantir l'obtention de bonnes performances (Mueller \& Dweck, 1998). Ces résultats vont dans le 
sens des résultats généralement obtenus dans la littérature où sont décrits les effets positifs d'une motivation orientée vers la maitrise sur le niveau de motivation intrinsèque (Cury et al., 1996 ) mais également sur l'utilisation de stratégies d'apprentissage adaptées et efficaces (Ames \& Archer, 1988) et donc sur l'obtention de meilleures performances scolaires (Midgley \& Urdan, 2001).

A noter tout de même que la condition évaluative orientée vers la performance n'est pas nuisible aux apprentissages puisqu'elle permet également aux élèves de progresser au cours des 5 essais. Simplement, cette progression est moins marquée et les performances restent moindres que dans la condition orientée vers la maîtrise. De la même manière, les élèves de cette condition semblent avoir adopté, pour ce type de tâche, une motivation orientée vers la performance : en choisissant majoritairement pour le prochain exercice une tâche visiblement très simple, on peut penser qu'ils privilégient ainsi des situations déjà maîtrisées, ne les mettant pas en danger et leur permettant, à défaut d'effectuer de nouveaux apprentissages, d'obtenir de bonnes performances. Ces résultats semblent aller dans le sens des résultats habituellement obtenus dans la littérature. On sait en effet que la poursuite de buts de performances permet dans certaines conditions l'obtention de bonnes performances (Darnon et al., 2009 ; Harackiewicz et al., 2002). En fait, il semble qu'orienter l'attention des individus sur leurs résultats peut être relativement favorable à ces résultats mais que cela n'encourage pas ces mêmes individus à effectuer des traitements profonds de la tâche (Midgley et al., 2001 ; Darnon, Muller, Schrager, Pannuzzo, \& Butera, 2006).

\section{Le rôle du temps : de la situation au climat}

35 D’après nos résultats, le rôle bénéfique de la condition évaluative orientée vers la maîtrise semble se renforcer avec le temps : plus les enfants sont exposés aux consignes campant le climat évaluatif, plus l'écart se creuse entre les performances d'une condition à l'autre. Ces résultats poussent donc à envisager le rôle des contextes évaluatifs sur un plus long terme qu'une simple induction situationnelle : il est en effet possible que les effets obtenus dans cette étude soient démultipliés s'ils étaient observés à l'échelle d'un laps de temps plus important (une année scolaire). L'idée de l'importance du climat installé à long terme dans une classe sur la motivation et les performances des élèves n'est pas nouvelle et a fait l'objet de multiples études, pour la plupart corrélationnelles, mettant en relation les pratiques des enseignants et les représentations/comportements des élèves (Epstein, 1988; Ames, 1992 a et b; Ames \& Ames, 1984 ; Ames \& Archer, 1988 ; Ames \& Maehr, 1989). Ces études s'intéressent à de multiples facteurs dont il est difficile de démêler chacun des effets propres. L'objectif d'une future étude serait plutôt ici de mettre en place une étude longitudinale reprenant le protocole de celle-ci sur un temps plus long (une année scolaire par exemple) de manière à mesurer les effets des différents contextes évaluatifs sur les comportements et performances des élèves.

\section{Noter ou ne pas noter ... est-ce la question?}

D'un point de vue scientifique, cette question n'est sans doute pas primordiale. En effet, certains travaux montrent qu'avec une note, un contexte évaluatif peut être plus ou moins menaçant selon qu'il est orienté vers la maîtrise ou la performance (Souchal, Toczek, Darnon, Smeding, Butera \& Martinot, 2013). Dans la même veine, le premier 
des points forts de cette étude est de répliquer les effets bénéfiques d'un climat orienté vers la maîtrise auprès d'enfants plus jeunes que la population habituellement observée, composée très souvent d'étudiants. Le second est de montrer que même dans un contexte où les enfants ne sont pas notés, il est possible, par les cibles de comparaison invoquées, de rendre les contextes plus ou moins bénéfiques à l'apparition de certaines motivations, et à la production de plus ou moins bonnes performances. En effet, dans la littérature, le climat de classe orienté vers la performance est très souvent induit par l'utilisation d'une évaluation normative (Kaplan \& Maehr, 2007). Cette étude montre donc que, plus que le recours ou non à la note, c'est la finalité de l'évaluation qui importe et qui impacte plus ou moins les performances des élèves. Ainsi peut-on dire que les climats plus orientés vers la maîtrise, mettant plus en exergue la mission formatrice de l'école seraient ceux qui, indépendamment de l'usage ou non des notes, seraient les plus favorables aux apprentissages et aux performances. De même, les climats de performance seraient ceux qui, renforçant la compétition et l'aspect sélectif du système scolaire, et toujours indépendamment de l'usage ou non des notes, seraient les moins bénéfiques. L'hypothèse serait que ces effets se renforcent toujours plus en fonction du temps d'exposition des élèves à un même climat. Elle devra être mise à l'épreuve des faits, en observant les performances des élèves sur une, voire plusieurs années. Plusieurs perspectives pourraient ainsi être pensées avec la volonté d'étudier les effets d'une alternance de climats d'une année à l'autre. De tels travaux pourraient offrir des éléments de compréhension aux adaptations demandées aux élèves lors du passage d'une classe à l'autre ou d'un établissement à l'autre.

\section{BIBLIOGRAPHIE}

Ames, C. (1984). Competitive, cooperative, and individualistic goal structures: A cognitive motivational analysis. In R. Ames \& C. Ames (Eds.), Research on motivation in education: Student motivation (Vol. 1, pp. 177-208). New York: Academic Press.

Ames, C. (1992a). Achievement goals and the classroom motivational climate. In D. H. Schunk \& J. L. Meece (Eds.), Student perceptions in the classroom (pp. 327-347). NJ: Erlbaum.

Ames, C. (1992b). Classrooms: Goals, structures, and student motivation. Journal of Educational Psychology, 84, 261-271. doi:10.1037/0022-0663.84.3.261

Ames, C., \& Ames, R. (1984). System of student and teacher motivation: Toward a qualitative definition. Journal of Educational Psychology, 76, 535-556. doi:10.1037/0022-0663.76.4.535

Ames, C., \& Archer, J. (1988). Achievement goals in the classroom: Student's learning strategies and motivation process. Journal of Educational Psychology, 80, 260-267.

doi: $10.1037 / 0022-0663.80 .3 .260$

Ames, C.A., \& Maehr, M. (1989). Home and school corporation in social and motivational development. Research funded by the Office of Special Education and Rehabilitative Services. Technical Report.

Anderman, E. M., Griesinger, T., \& Westerfield, G. (1998). Motivation and cheating during early adolescence. Journal of Educational Psychology, 90(1), 84-93. 
Anderman, E. M., \& Midgley, C. (1997). Changes in personal achievement goals and the perceived classroom goal structures across the transition to middle level schools. Contemporary Educational Psychology, 22, 269-298. doi : 0361-476X/97

Butera, F., Buchs, C. \& Darnon, C. (2011). L'évaluation, une menace? Paris: PUF

Butler, R. (1987). Task-involving and ego-involving properties of evaluation: Effects of different feedback conditions on motivational perceptions, interest, and performance. Journal of Educational Psychology, 79, 474-482. doi: 10.1037/0022-0663.79.4.474

Butler, R. (1993). Effects of task- and ego-achievement goals on information seeking during task engagement. Journal of Personality and Social Psychology, 65, 18-31.

Butler, R., \& Nissan, M. (1986). Effects of No Feedback, Task-Related Comments, and Grades on Intrinsic Motivation and Performance. Journal of Educational Psychology, 78, 210-216. doi : 10.1037//0022-0663.78.3.210

Crahay, M. (1996). Peut-on lutter contre l'échec scolaire ? Bruxelles, Belgique : De Boeck.

Cury, F., Biddle, S., Famose, J.-P., Goudas, M., Sarrazin, P., \& Durand, M. (1996). Personal and Situational Factors Influencing Intrinsic Interest of Adolescent Girls in School Physical Education: a structural equation modeling analysis. Educational Psychology, 16, 305-315. doi :

10.1080/0144341960160307

Darnon, C., Dompnier, B., Delmas, F., Pulfrey, C., \& Butera, F. (2009). Achievement goal promotion at university: Social desirability and social utility of mastery and performance goals. Journal of Personality and Social Psychology, 96(1), 119-134. doi:10.1037/a0012824

Darnon, C., Dompnier, B. \& Marijn Poortvliet, P. (2012), Achievement Goals in Educational Contexts: A Social Psychology Perspective. Social and Personality Psychology Compass, 6: 760771.

Darnon, C., Muller, D., Schrager, S. M., Pannuzzo, N., \& Butera, F. (2006). Mastery and performancegoals predict epistemic and relational conflict regulation. Journal of Educational Psychology, 98, 766-776. doi :10.1037/0022-0663.98.4.766

Dubet, F., \& Martucelli, D. (1988). Dans quelle société vivons-nous ? Paris, France : Seuil. Duru-Bellat, M. (2009). Le Mérite contre la Justice, Paris, France : Presses de Sciences Po.

Elliot, A.J., \& Dweck, C.S. (1988). Goals: An approach to motivation and achievement. Journal of Personality and Social Psychology, 54, 5-12. doi: 10.1037/0022-3514.54.1.5

Epstein, J.L. (1988). Effective schools or effective students: Dealing with diversity. Dans R. Haskins \& D. Mac-Rae (Eds.), Policies for America's public schools: Teacher equity indicators (pp. 89-126). Norwood, PA: Ablex.

Hadji, C. ( 2015). L'évaluation à l'école. Paris: Nathan.

Hattie, J., \& Timperley, H. (2007). The power of feedback. Review of Educational Research, 77, 81-112. Huguet, P., \& Régner, I. (2007). Stereotype threat among school girls in quasi-ordinary classroom circumstances. Journal of Educational Psychology, 99, 545-560. doi :10.1037/0022-0663.99.3.545

Kaplan, A., \& Maehr, M. (2007). The contributions and prospects of goal orientation theory. Educational Psychology Review, 19, 141-184. doi :10.1007/s10648-006-9012-5

Meece, J.L., Anderman, E.M., \& Anderman, H.L. (2006). Classroom goal structure, student motivation, and academic achievement. Annual Review of Psychology, 57, 487-503. doi : 10.1146/ annurev.psych.56.091103.070258 
Midgley, C., \& Urdan, T. (1995). Predictors of middle school students' use of selfhandicapping strategies. Journal of Early Adolescence, 15, 389-411. doi : 10.1177/0272431695015004001

Midgley, C., \& Urdan, T. (2001). Academic self-handicapping and achievement goals : A further examination. Contemporary Educational Psychology, 26, 61-75. doi : 10.1177/0272431695015004001

Ministère de l'Education Nationale (2013). La notation et l'évaluation des élèves éclairées par des comparaisons internationales. http://cache.media.education.gouv.fr/file/2013/98/7/RapportIGEN-2013-072_274987.pdf

Mons, N., Toczek, M-C., West, A., \& Barkous, S. (2014). L'évaluation des élèves par les enseignants dans la classe et les établissements : réglementation et pratiques. Une comparaison internationale dans les pays de l'OCDE. Décembre 2014. CNESCO

Mueller, C.M., \& Dweck C.S. (1998). Praise for intelligence can undermine children's motivation and performance. Journal for Personality and Social Psychology, 75, 33-52. doi :

10.1037/0022-3514.75.1.33

Ntoumanis, N. (2001). A self-determination approach to the understanding of motivation in physical education. British Journal of Educational Psychology, 71, 225-242. doi :

10.1348/000709901158497

Rawsthorne, L.J., \& Elliott, A.J. (1999). Achievement goals and intrinsic motivation : A metaanalytic review. Personality and Social Psychology Review, 3, 326-344. doi :10.1207/

s15327957pspr0304_3

Sarrazin, P., Famose, J-P., \& Cury, F. (1995). But motivationnel, habileté perçue et sélection du niveau de difficulté d'une voie en escalade. STAPS : Revue internationale des sciences du sport et de l'éducation physique, 38, 49-61.

Souchal, C., Toczek, M-C., Darnon, C., Smeding, A. Butera, F. \& Martinot, D. (2013). Assessing Does Not Mean Threatening : Assessment as a key determinant of girls' and boys' performance in a science class. British Journal of Educational Psychology, 84, 125-136. doi :10.1111/bjep.12012

\section{ANNEXES}

Annexe 1 : Tableau synthétique d'aide à la lecture de la revue de question, partie Évaluation et type de motivation 


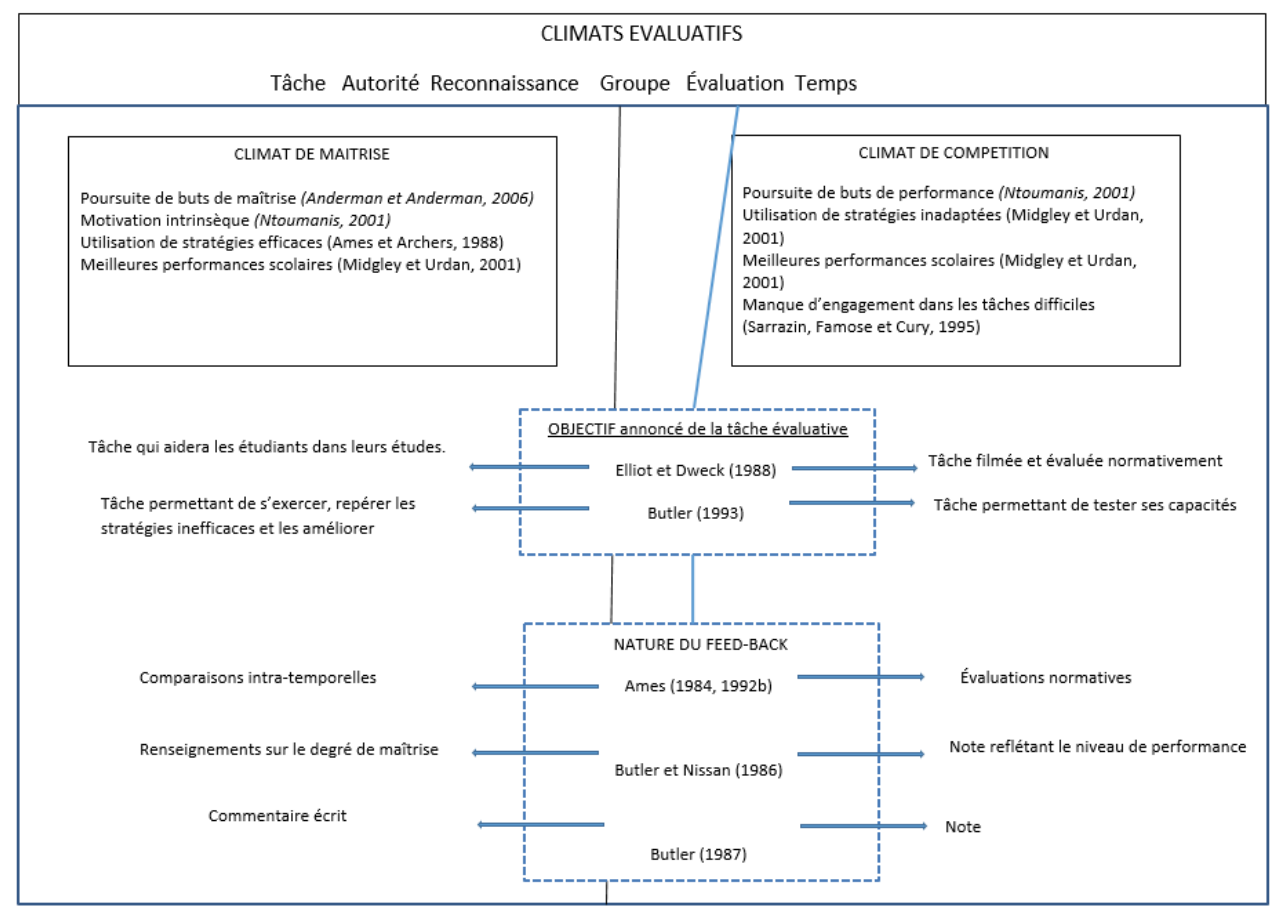

\section{RÉSUMÉS}

Contexte: Cette étude part des polémiques récurrentes que l'école connaît au sujet de l'évaluation des élèves. Elle met en évidence la différence d'impact du contexte évaluatif sur les performances des élèves.

Objectif: Elle s'intéresse aux effets de différents contextes évaluatifs sur les performances d'élèves âgés de 9 ans lors d'un apprentissage.

Méthode: Tous les participants sont soumis à une séquence d'apprentissage d'une figure géométrique en 5 essais. Certains sont placés dans un contexte évaluatif orienté vers la performance alors que les autres sont placés dans un contexte orienté vers la maîtrise.

Résultats: Les résultats montrent que les élèves placés dans un contexte orienté vers la performance ont de moins bons résultats et apprennent moins vite que les élèves placés dans un contexte évaluatif orienté vers la maîtrise. Cet effet semble s'accentuer avec le temps d'exposition à ces différents climats.

Conclusion : Ces résultats sont discutés avec une visée applicative : ils suggèrent en effet que les débats souvent centrés sur les procédés évaluatifs eux-mêmes devraient plutôt envisager les climats de classe dans lesquels ces évaluations sont instaurées.

Background: The starting point for this study is the recurrent debate surrounding pupil assessment at school. This debate highlights the differences in the ways pupil achievement is impacted by the context.

Aims and Sample: Our study focuses on the effects different evaluative contexts (performance oriented versus mastery oriented contexts) shave on learning for some 4 th grade students.

Methods: All the pupils take part in a learning sequence, during which they are allowed five tries at reproducing a geometric figure. Some pupils work in a performance-oriented assessment context, whereas others work in a mastery-oriented assessment context

Results: The results show that the pupils in the performance- oriented assessment context do not get such good results and do not learn as fast as the pupils in the mastery- oriented 
assessment context. The longer the pupils are exposed to these different contexts, the greater these effects.

Conclusions: Our discussion of these findings aims to show how they can be applied: it is suggested that the discussions which often focus on the evaluative procedures themselves should focus to considering the classroom climates in which assessments are carried out.

\section{INDEX}

Mots-clés : évaluation, contexte, buts de maitrise, buts de performance, performances

Keywords : evaluation, context, performance goals, mastery goals, performance

\section{AUTEURS}

\section{CARINE SOUCHAL}

Post-doctorante, Membre associé à l'unité de recherche ACTé, EA 4281, Clermont Université, Université Blaise-Pascal, Clermont-Ferrand, France

\section{MARIE-CHRISTINE TOCZEK}

Professeure des Universités, unité de recherche ACTé, EA 4281, Clermont Université, Université Blaise-Pascal, Clermont-Ferrand, France 